\title{
Periodic upward migration model for intermediate-depth earthquakes in Vrancea, Romania
}

\author{
Nobuo Hurukawa ${ }^{1}$ and Masajiro Imoto ${ }^{2}$ \\ ${ }^{1}$ International Institute of Seismology and Earthquake Engineering (IISEE), Building Research Institute, Tsukuba, Ibaraki 305-0802, Japan \\ ${ }^{2}$ National Research Institute for Earth Science and Disaster Prevention (NIED), Tsukuba, Japan
}

(Received February 16, 2009; Revised January 27, 2010; Accepted February 22, 2010; Online published July 9, 2010)

\begin{abstract}
Vrancea intermediate-depth earthquakes with a depth range of 60-160 km occur frequently. By using data from ROMPLUS catalog, we analyze earthquakes with $M \geq 7.0$ that occurred during 1500-2000. We propose a periodic upward migration (PUM) model for these earthquakes. According to this model, (1) active zones at the bottom of seismic regions migrate upward with the velocity $V$, with the activity starting from the year $T_{S}$ and, (2) this migration repeats every $T_{P}$ years, and at a given depth, seismic active period continues for $T_{1}$ years. We test the model using the likelihood function and compare the model with a uniform Poisson model. A simulation using the bootstrap method indicates that the PUM model for $M \geq 7.5$ and $M \geq 7.3$ earthquakes is statistically signi cant at the $5 \%$ level and is better than the Poisson model. We propose the following regularity for Vrancea $M 7$ intermediate-depth earthquakes. Three earthquakes occur at a depth of 140-160 km, 110-140 km, and 80$110 \mathrm{~km}$ at the beginning, at the middle, and at the end of each century, respectively. This phenomenon repeats every century. The next $M 7$ earthquake is expected to occur at a depth of 140-160 km soon.
\end{abstract}

Key words: Intermediate-depth earthquake, Vrancea earthquake, earthquake prediction, likelihood function, migration.

\section{Introduction}

Intermediate-depth earthquakes with a magnitude $(M)$ of 7 or greater frequently occur in the Vrancea region of Romania and cause severe damages in Romania as well as the neighboring countries. In particular, Bucharest in Romania has suffered severe damages due to earthquakes since this city is located at an epicentral distance of approximately 150 km (Fig. 1(a), e.g. Wenzel et al., 1999; Frohlich, 2006). For predicting earthquakes and mitigating seismic disasters, it is necessary to understand the mechanisms of the occurrence of earthquakes. This information will help us in taking the necessary earthquake countermeasures.

By studying the behavior of historical earthquakes, Purcaru (1974, 1979) and Enescu et al. (1974) successfully predicted the 1977 earthquake (M 7.5) and predicted the next one that would occur at the beginning of this century. However, their proposed hypothesis lacked a seismological background, and its statistical validity was not examined.

On the other hand, using a stress-release model and data obtained over the last 500 years, Imoto and Hurukawa (2006) concluded that the probability of an $M \geq 7.0$ earthquake occurring in the Vrancea region in a 5-year period would exceed $40 \%$ by 2010 . This implies that an earthquake can be expected to occur soon.

Furthermore, by relocating $M \geq 6.0$ earthquakes that have occurred since 1934 using the modi ed joint hypocen-

Copyright (C) The Society of Geomagnetism and Earth, Planetary and Space Sciences (SGEPSS); The Seismological Society of Japan; The Volcanological Society of Japan; The Geodetic Society of Japan; The Japanese Society for Planetary Sciences; TERRAPUB. ter determination (MJHD) method, Hurukawa et al. (2008) proposed a seismic gap at a depth of $140-160 \mathrm{~km}$, where the next $M 7$ earthquake may be expected to occur (Fig. 1(b)).

In this study, by analyzing the past 500-year data on earthquakes, we propose a "periodic upward migration (PUM) model;" according to this model, the occurrence of $M 7$-class ( $M 7$ ) earthquakes is found to shift from deep to shallow segments of seismic regions. Further, we statistically verify this model by using the likelihood function and bootstrap simulation. Furthermore, we examine the seismic characteristics of Vrancea earthquakes in detail and elucidate how these occur.

Although many studies on migration of earthquakes were done, many of them were on migration of epicenters of shallow earthquakes (e.g., Utsu, 1999). Concerning deep earthquakes, studies were mainly related to relationship between deep and shallow earthquakes (e.g., Utsu, 1975; Mogi, 1987). There are some studies on depth migration of deep earthquakes (e.g., Mogi, 1973, 1987). Mogi (1973) found that the seismic activity trends to progress in time from a shallow to a deep region within a descending lithosphere in the Mariana and Tonga arcs and the rate of migration is estimated at about $50 \mathrm{~km}$ per year. Mogi (1987) found another downward migration in the central part of Japan, in which seismic activity gradually migrated downward and the Tonankai earthquake occurred when it reached its limit (a depth approximately $70 \mathrm{~km}$ ). Since the direction of these migration phenomena is downward and is different from ours, that is upward, the mechanism of the migration may differ each other. 
(a)

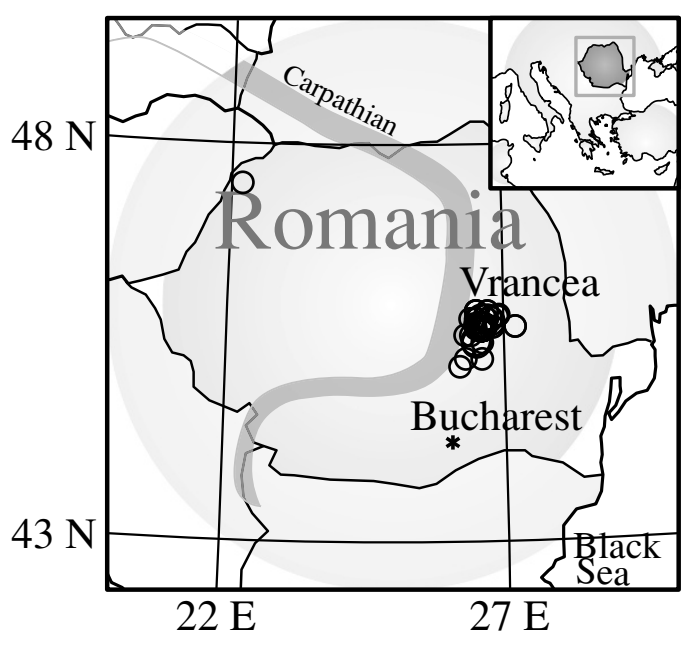

(b)

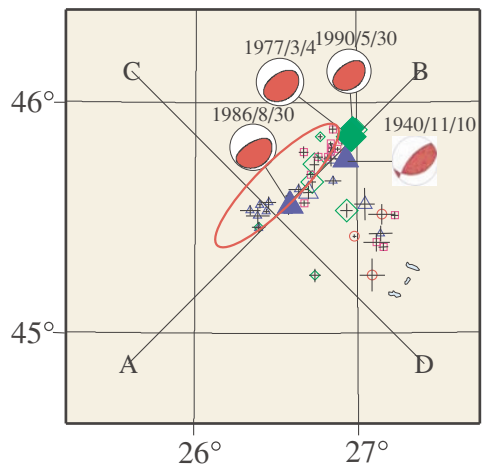

Magnitude

$\begin{array}{lllll}5 & 6 & 7 & 8\end{array}$

Depth

40
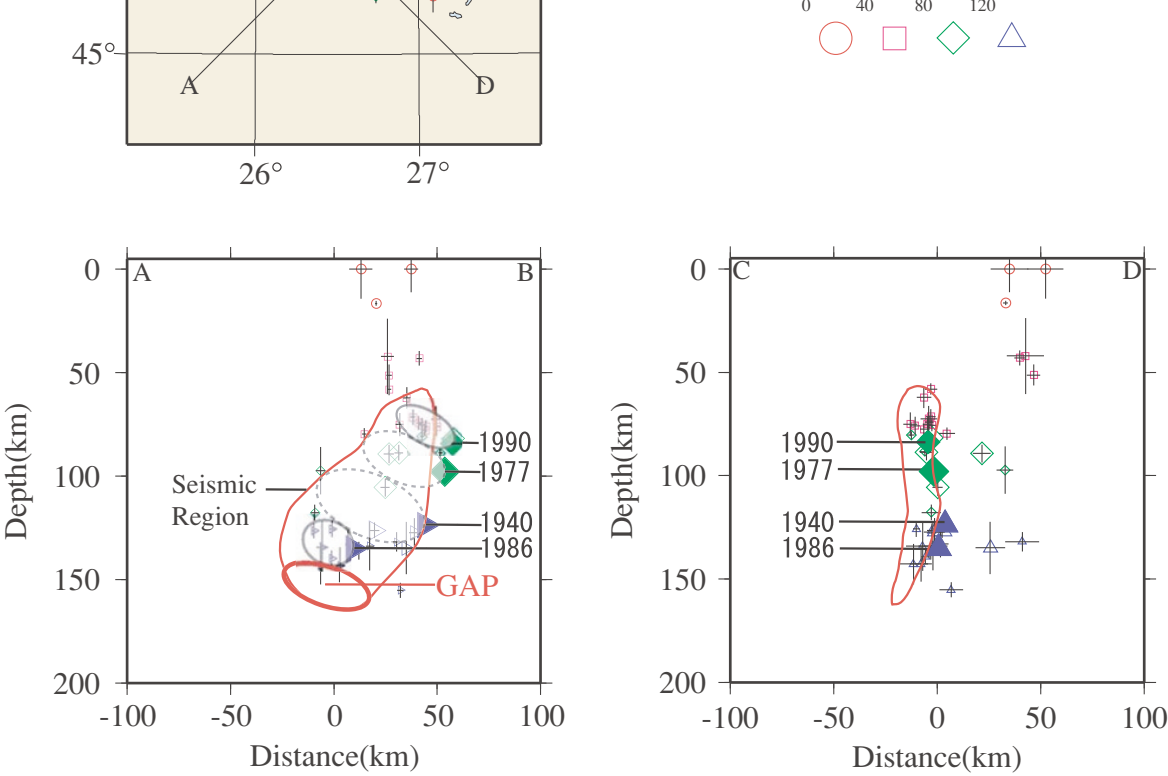

Fig. 1. Location of Vrancea intermediate-depth earthquakes. (a) Earthquakes with $M \geq 6.0$ earthquakes that occurred during $1500-2000$ in Romania (data from ROMPLUS catalog). Almost all earthquakes are intermediate-depth earthquakes in Vrancea. (b) Relocated $4 M \geq 6.9$ earthquakes and proposed seismic gap (obtained from figure 6(b) shown in Hurukawa et al., 2008).

\section{Original Data}

We have used data from the ROMPLUS catalog for the analysis; this catalog is a Romanian earthquake catalog compiled by Oncescu et al. (1999) and is currently maintained by the National Institute for Earth Physics (NIEP). This catalog (ROMPLUS), in which earthquake parameters were adopted by Costantinescu and Marza (1980), has been used in Imoto and Hurukawa (2006), too. Accord- ing to Oncescu et al. (1999), the best feature of this catalog is that it is (1) up-to-date, (2) complete, (3) homogeneous both in the location procedure and in the magnitude scale, and (4) easily accessible. ROMPLUS contains information on earthquakes that have occurred in Romania since 984. Figure 2 shows the magnitude vs. time $(M-T)$ diagram of these earthquakes; the largest earthquake among them is the M 7.9 earthquake that occurred in 1802. 
Table 1. List of earthquakes with $M \geq 7.0$ (ROMPLUS by Oncescu et al., 1999).

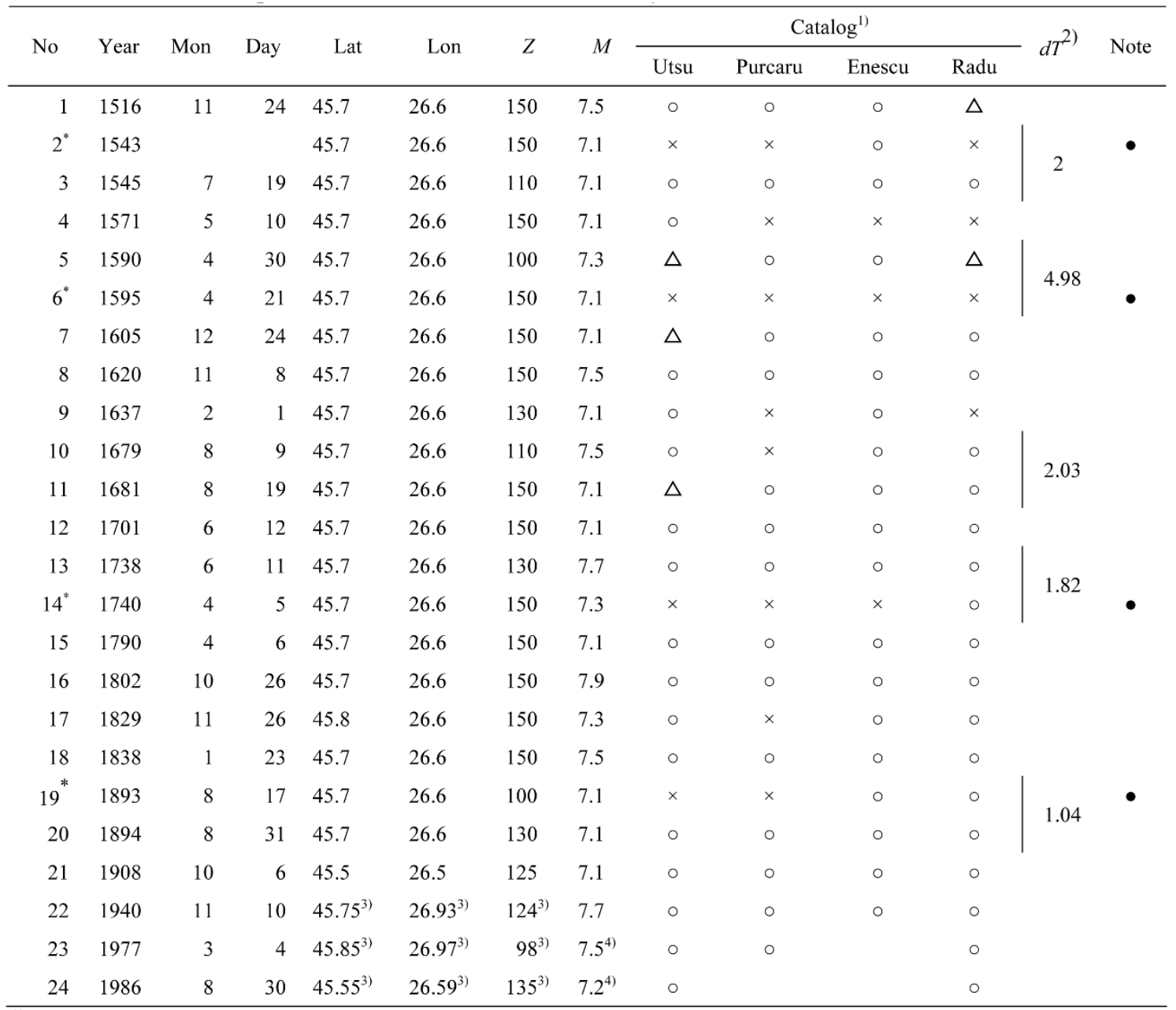

${ }^{1)}$ Catalog: Following catalogs are used.

Utsu: Utsu $(1990,1992)$

Purcaru: Purcaru (1979)

Enescu: Enescu et al. (1974)

Radu: Lungu and Aldea (2001)

O: Same date. $\triangle$ : Different date. $\times$ : No description.

${ }^{2)} d T$ : Time difference in year between two earthquakes of which interval are less than 5 years.

${ }^{3)}$ Hypocenters by Hurukawa et al. (2008)

4) $M w$ by Harvard University (Dziwonski et al. (1981) and later updates).

${ }^{*}$ and $\bullet$ : Fake $M 7$ earthquakes

We analyze intermediate-depth earthquakes occurring in the Vrancea region with $M \geq 7.0$ and a focal depth of $Z \geq 60 \mathrm{~km}$; the selection criteria for these earthquakes are the same as those reported in Imoto and Hurukawa (2006). Furthermore, we use the relocated hypocenter parameters of earthquakes that have occurred after 1940 for the analysis, as described in Hurukawa et al. (2008). We adopt the moment magnitude scale $\left(M_{\mathrm{w}}\right)$ reported by Harvard University (Dziwonski et al., 1981; later updates) to represent the magnitude of earthquakes that have occurred after 1977.

As shown in Fig. 2, it is clear that $M \geq 7.0$ earthquakes have constantly occurred since the year 1000 , except during $1250-1450$. We, therefore, use the data for the period 1500-
2000 from the catalog; the details of earthquakes that occurred during this period are listed in Table 1 . This dataset is called Dataset 1, in which 24 earthquakes are included. It should be noted that no $M \geq 7.0$ earthquakes have occurred since 2000 .

\section{Periodic Upward Migration (PUM) Model}

Purcaru $(1974,1979)$ has proposed a model for earthquakes in which earthquakes exhibit 3 seismic active periods in each century characterized by "quasicycles." Hurukawa et al. (2008) have proposed that seismic regions can be divided into 3 major segments: shallow (depth: 80$110 \mathrm{~km}$ ), midst (depth: 110-140 km), and deep (depth: 


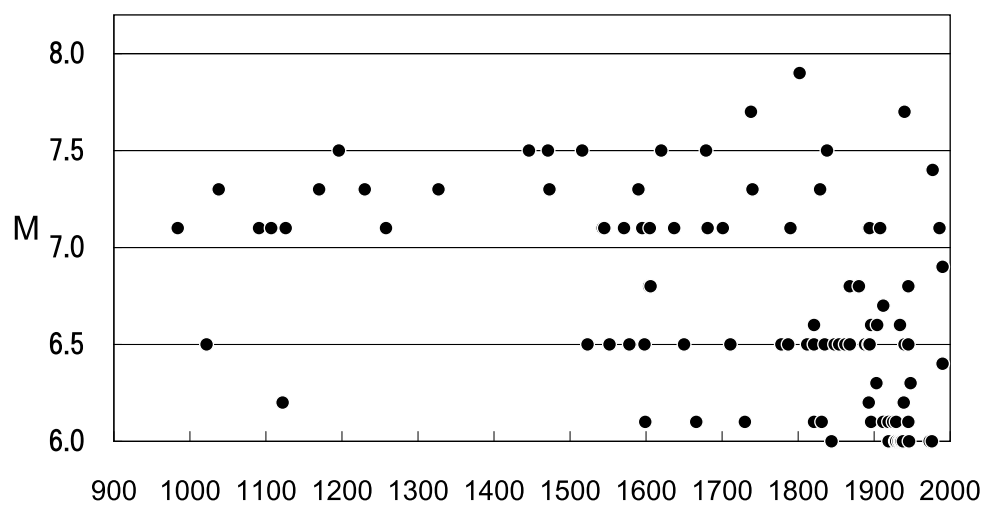

Year

Fig. 2. $M-T$ diagram for earthquakes with $M \geq 6.0$ and $Z \geq 60 \mathrm{~km}$ obtained from ROMPLUS (Oncescu et al., 1999).

a Year for Each Century

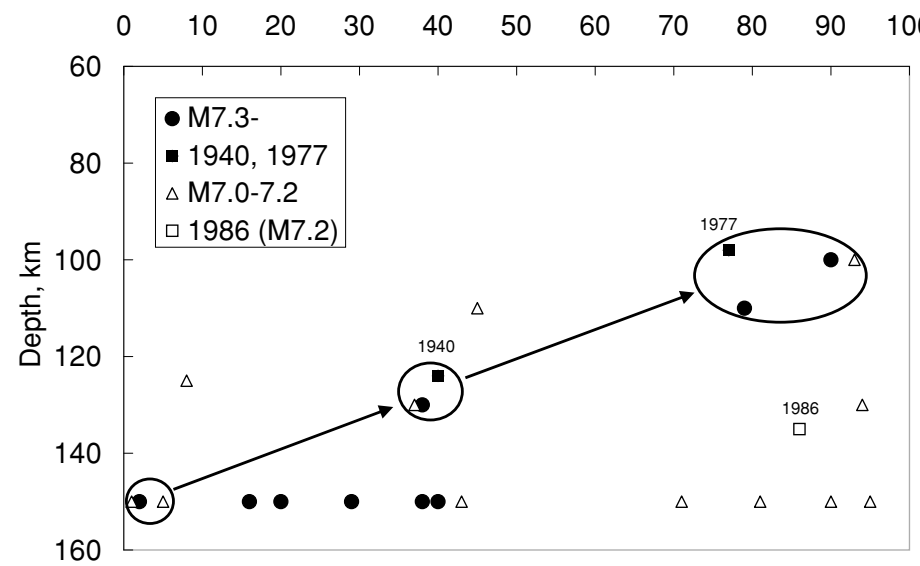

b Year for Each Century

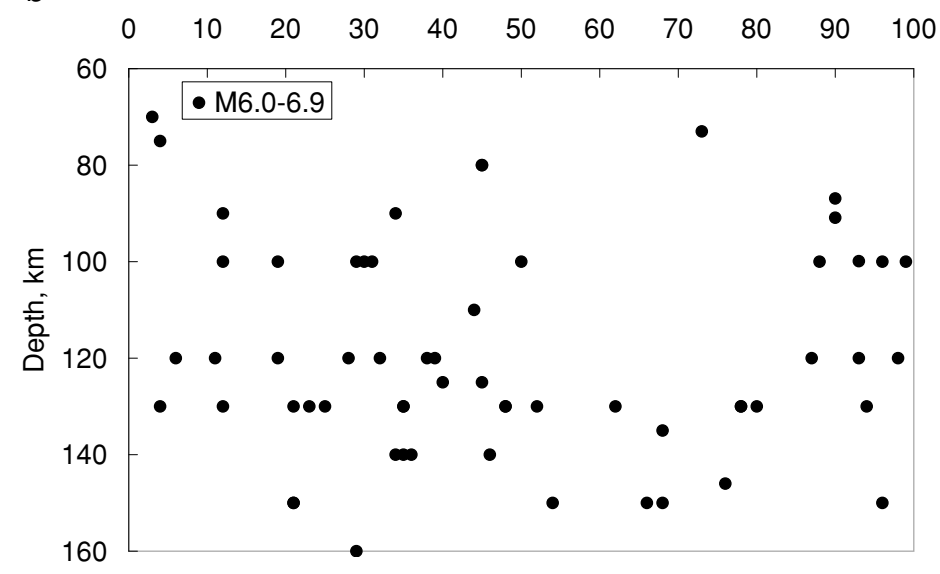

Fig. 3. Relationship between focal depth and year of occurrence of large earthquakes for each century that occurred during 1500-2000. Data is obtained from ROMPLUS catalog (Oncescu et al., 1999). (a) $M \geq 7.0$ earthquakes. The squares indicate the 1940, 1977, and 1986 earthquakes relocated in Hurukawa et al. (2008). The large solid circles and small open triangles indicate $M \geq 7.3$ and $7.0 \leq M \leq 7.2$ earthquakes, respectively. The open ellipses indicate main activities at the 3 seismic active periods and the arrows indicate the migration of seismicity from deep to shallow segments in seismic regions in each century. (b) Earthquakes with $6.0 \leq M \leq 6.9$.

$140-160 \mathrm{~km})$. On the basis of these models, we propose the following regularity for Vrancea intermediate-depth earthquakes. The first $M 7$ earthquake occurs in a deep segment at the beginning of each century, the second $M 7$ earthquake occurs in a midst segment in the middle of each century, and the third $M 7$ earthquake occurs in a shallow segment at the end of each century. Note that no $M 7$ earthquake occurs in the region above the shallow segment (depth: 60$80 \mathrm{~km}$ ) because of its shorter length. We call this model the periodic upward migration (PUM) model. To test the 


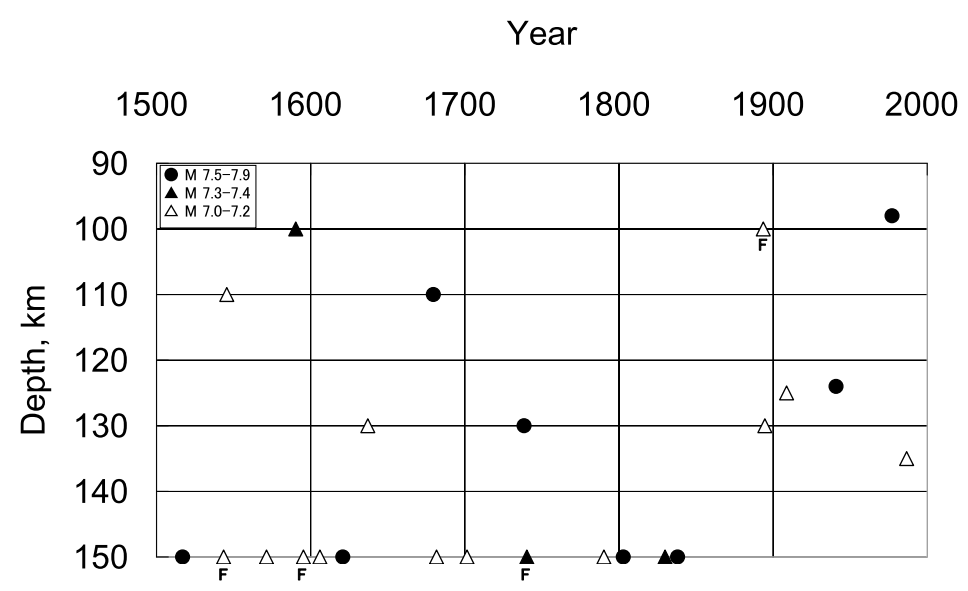

Fig. 4. Relationship between years of occurrence and focal depths of earthquakes (Table 1). The large solid circles, small solid triangles, and open triangles represent $M \geq 7.5,7.3 \leq M<7.5$, and $7.0 \leq M<7.3$ earthquakes, respectively. F: Fake $M 7$ earthquakes (refer text and Table 1).

proposed model, we plot the focal depth of $M 7$ - and $M$ 6class earthquakes against the year of their occurrence for each century as shown in Figs. 3(a) and 3(b), respectively. Since the focal depths of old earthquakes have been estimated from seismic intensity distributions of these earthquakes, these focal depths may be not quite accurate. However, the focal depths of half of the $M$ 7-class earthquakes, especially $M \geq 7.3$ earthquakes, exhibit a clear dependence on seismic active periods for each century. The scatter in the data on $7.0 \leq M \leq 7.2$ earthquakes can be attributed to (1) the inaccurate focal depths and (2) actual occurrences of earthquakes like the 1986 (M 7.2) earthquake. On the other hand, no clustering is detected in the case of $M$ 6-class earthquakes, which appeared to occur randomly. These results support the proposed model with respect to the main trends in the seismic regime evolution in the Vrancea region. From the hypocenter relocation image obtained by Hurukawa et al. (2008) and the observed periodicities of earthquakes in the seismic regime, it is highly probable that the next strong shock will be generated by around 2010 in the deep segment (depth: 140-160 km) of the Vrancea focal volume. This migration phenomenon is investigated in detail in this study.

\section{Statistical PUM Model}

Figure 4 shows the focal depth of all earthquakes against the year of their occurrence. It should be noted that no $M \geq 7.0$ earthquakes have occurred in the Vrancea region outside a depth range of 90-150 km. The PUM model could explain the behavior of many of the $M \geq 7.0$ earthquakes (particularly $M \geq 7.3$ earthquakes) that have occurred during the past 500 years, as shown in Fig. 3(a).

We have proposed a statistical model of the PUM model, as shown in Fig. 5(a). This model is based on a Poisson process with two rates cyclically altered, which comprises active zones (indicated by the hatched region in Fig. 5(a)) and inactive zones: according to this model, active zones at the bottom of seismic regions migrate upward. We assume that the seismicity of the either zones conforms to a uniform Poisson model. The Poisson rate of the seismicity in active zones is greater than that in inactive zones. According to the proposed model (1) active zones at the bot- tom of seismic regions migrate upward with the velocity $V$ (km/year), with the activity starting from the year $T_{S}$; (2) this migration phenomenon repeats every $T_{P}$ years; and (3) at a given depth, seismic active period continues for $T_{1}$ years (indicated by the width of the hatched region in Fig. 5(a)). Since according to the proposed model the upward migration phenomenon repeats periodically, we can divide the period 1500-2000 indicated in Fig. 5(a) into several subperiods with an interval of $T_{P}$ years and superpose them, as shown in Fig. 5(b). This superposition simplifies the schematic diagram of the model.

Figure 5 and Table 2 show an example of the PUM model for $T_{P}=100$ years, which is an optimal solution of this model for $8 M \geq 7.5$ earthquakes, of which hypocenters and magnitudes are most reliable, shown in Table 1 . This solution is denoted as Solution 1.

$$
\begin{aligned}
T_{S} & =\text { year } 1502 \\
T_{P} & =100 \text { years } \\
T_{1} & =37 \text { years } \\
V & =0.95 \mathrm{~km} / \text { year }
\end{aligned}
$$

Let us examine if the statistical PUM model is better than the uniform Poisson model (according to which earthquakes occur at random time intervals and depths). We apply the method proposed by Imoto (1992) in which a change in seismicity is statistically examined using the likelihood function. Suppose the intensity of an earthquake occurring at a certain depth and certain time is represented by the intensity function $H(t, Z)$, where $t$ and $Z$ denote the time and focal depth, respectively.

Suppose $\lambda_{i}, S_{i}$ and $N_{i}$ denote the Poisson rate, the area in time-depth space, and the number of earthquakes that occurred in $S_{i}$, respectively ( $i=0,1,2$ ). The suffixes 0,1 , and 2 represent the values corresponding to the entire period (area), high-seismicity period (or active period), and lowseismicity period (or inactive period), respectively. Note that $S_{0}=S_{1}+S_{2}$ and $N_{0}=N_{1}+N_{2}$. Further, $L$ denotes the likelihood function. The suffixes 0 and 1 in the case of $L$ denote the uniform Poisson and PUM models, respectively. The expressions for $H(t, Z)$ for the PUM model can be 
Table 2. Results of best solutions for actual data during 1500-2000.

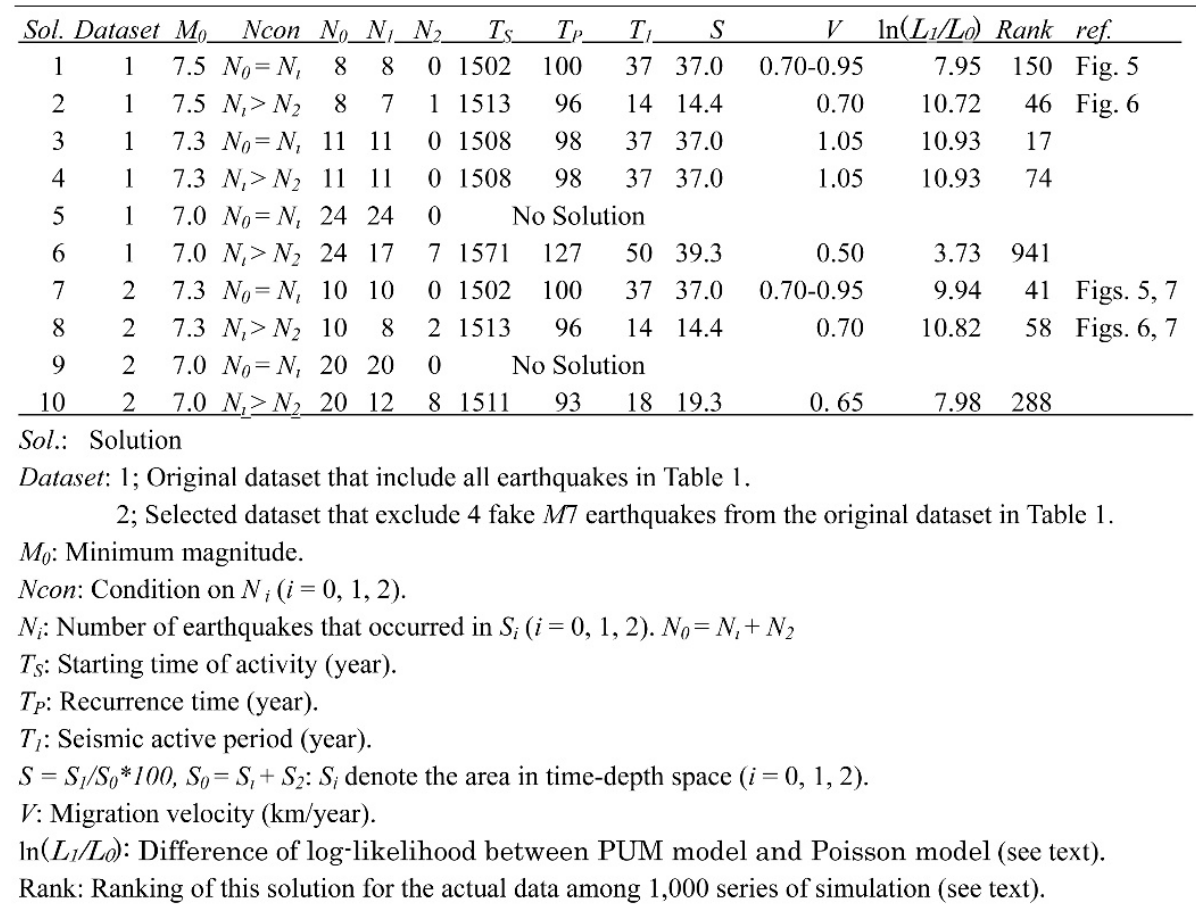

given as follows:

$$
H(t, Z)= \begin{cases}\lambda_{2} & 0<\bmod \left(\left(t-T_{S}\right), T_{P}\right) \leq d / V \\ \lambda_{1} & d / V<\bmod \left(\left(t-T_{S}\right), T_{P}\right) \leq T_{1}+d / V \\ \lambda_{2} & T_{1}+d / V<\bmod \left(\left(t-T_{S}\right), T_{P}\right) \leq T_{P}\end{cases}
$$

where $d=150-Z$ and $\bmod (x, y)$ is the remainder of $x / y$.

Therefore, the intensity functions and log-likelihood of the PUM model can be expressed as follows:

$$
\begin{aligned}
& \lambda_{0}=\frac{N_{0}}{S_{0}}, \lambda_{1}=\frac{N_{1}}{S_{1}}, \lambda_{2}=\frac{N_{2}}{S_{2}} \\
& \ln \left(L_{0}\right)=-N_{0}+N_{0} \ln \left(\lambda_{0}\right) \\
& \ln \left(L_{1}\right)=-N_{0}+N_{1} \ln \left(\lambda_{1}\right)+N_{2} \ln \left(\lambda_{2}\right)
\end{aligned}
$$

The difference between above two, $\ln \left(L_{1} / L_{0}\right)=\ln \left(L_{1}\right)-$ $\ln \left(L_{0}\right)$, will be used for the comparison of models. Note that $\ln \left(L_{1} / L_{0}\right)=7.95$ for Solution 1 above.

\section{Simulation of PUM Model}

The above mentioned results indicate a large $\ln \left(L_{1} / L_{0}\right)$ value in the case of the PUM model and the PUM model is better than the Poisson model. In this section, statistical signi cances of the optimal solution of the model for 8 earthquakes with $M \geq 7.5$ are examined by using the bootstrap method.

We have generated a time series of 8 earthquakes in which the observed relationship between the origin time and focal depth of the earthquakes were preserved. Firstly, we use the actual origin time of these 8 earthquakes. Then, we determine the focal depth of each earthquake as follows using a generated random number in order to preserve observed depth distribution. We select an earthquake from the 8 earthquakes by generating a random number by carrying out a simulation and use the focal depth of the selected earthquake as the depth of the simulated earthquake.

We have used the following conditions in our calculation:

1. Since the number of analyzed earthquakes is 8 at minimum, we allow 8 or less active periods during 500 years. This means that one or more earthquakes should occur at each active period on average. Therefore, $T_{P} \geq 63$ years.

2. The migration phenomenon is expected to be repeated 3 or more times during a period of 500 years. Therefore, $T_{P} \leq 166$ years.

3. The next activity should start after the front of the previous activity ends. Therefore, $T_{P}>60 / \mathrm{V}$.

4. Since $T_{S}$ is the year when the rst activity starts, $1500 \leq T_{S}<1500+T_{P}$. Therefore, $1500 \leq T_{S}<$ 1666.

5. Let the uncertainty in focal depth be denoted by $d Z$. Since the depth of historical earthquakes was given every $10 \mathrm{~km}$, we set $d Z=10 \mathrm{~km}$. The minimum $T_{1}$ value is set to the corresponding time interval. Since $V=d Z / d T, d T=d Z / V=10 / V$. Therefore, $T_{1} \geq 10 / V$. Since $V \leq 3.0 \mathrm{~km} /$ year, as explained below, the minimum $T_{1}$ value is 4 years.

6. Since we consider the migration of seismic activity, and not of seismic quiescence, active periods should be shorter than the inactive periods. Therefore, $T_{1}<$ $T_{P}-T_{1}$. That is, $T_{1}<T_{P} / 2$. Since $T_{P} \leq 166$, $T_{1} \leq 83$.

7. Since $T_{P} \leq 166$ years, the migration time should be less than or equal to 166 years. The migration time from $Z=150 \mathrm{~km}$ to $Z=90 \mathrm{~km}$ is $60 / V$ years so that $V>0.36 \mathrm{~km} /$ year. 
(a)

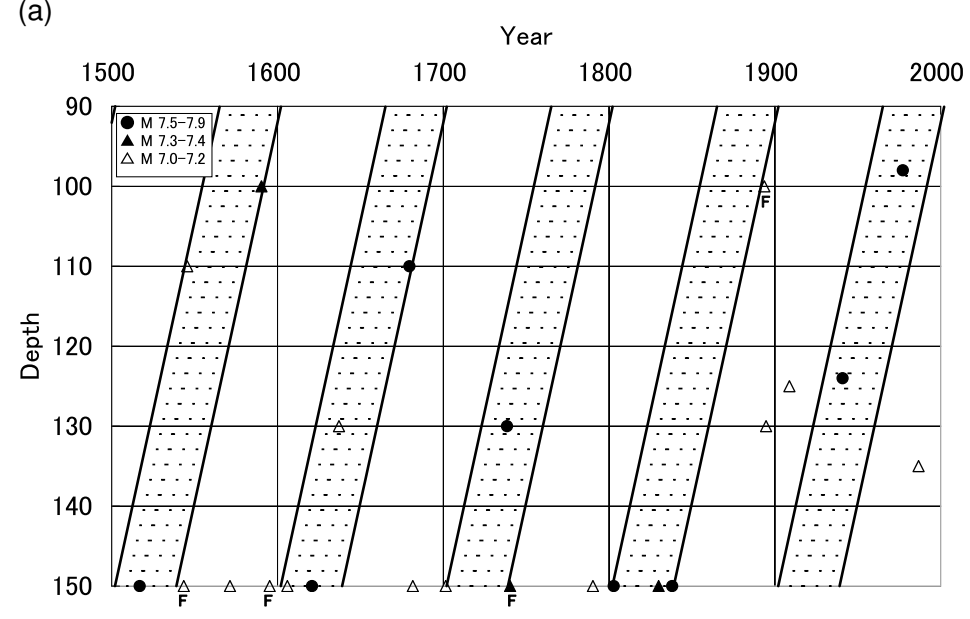

(b)
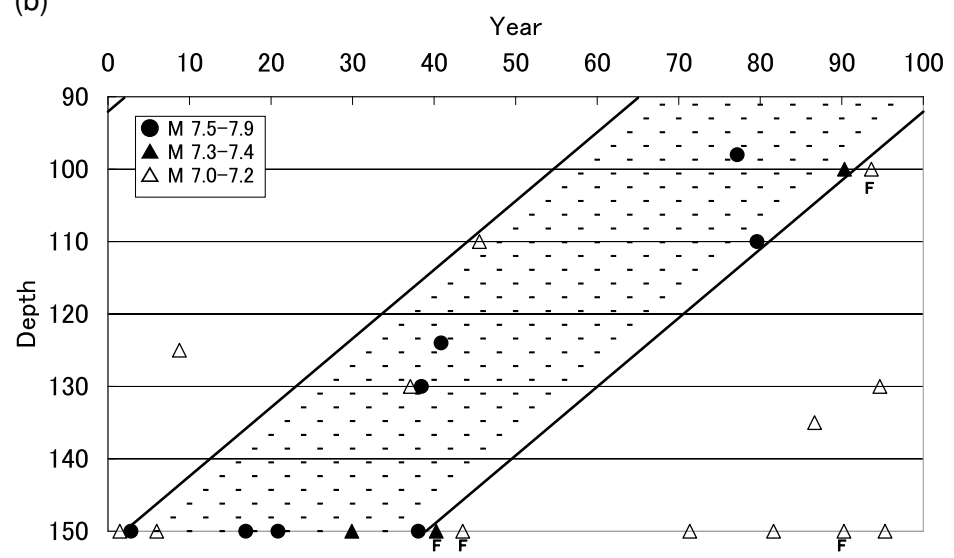

Fig. 5. (a) Statistical periodic upward migration (PUM) model for $M \geq 7.5$ earthquakes, in which $T_{P}=100$ years is shown as an example. (b) Superposed PUM model, in which the horizontal axis represents the year of each century. The hatched regions indicate high-seismicity periods. The symbols are the same as those used in Fig. 4. These are optimal solutions (Solutions 1 and 7 in Table 2) of the PUM model. F: Fake $M 7$ earthquakes shown in Table 1 (see text).

8. We consider the depth migration model, and not a model in which earthquakes occur at all depths. Hence, we set the minimum migration time as 20 years. Therefore, $V \leq 3.0$.

The ranges of the 4 parameters are summarized as follows:

$$
\begin{aligned}
& 63 \leq T_{P} \leq 166 \text { and } 60 / V<T_{P} \\
& 1500 \leq T_{S}<1500+T_{P} \leq 1666 \\
& 4 \leq T_{1}<T_{P} / 2 \leq 83 \\
& 0.4 \leq V \leq 3.0
\end{aligned}
$$

The intervals of the parameters $T_{P}, T_{S}$, and $T_{1}$ are 1 year, and the interval of $V$ is $0.05 \mathrm{~km} /$ year.

Furthermore, we consider a constraint on the number of earthquakes. Since we consider the migration of seismic activity, earthquakes occurred in the active periods should be greater than those in the inactive periods like time period explained in Item 6. Therefore, $N_{1}>N_{2}$. However, we also consider the extreme case that all earthquakes occurred in the active periods. That is $N_{0}=N_{1}$.

Calculating the difference of log-likelihood, $\ln \left(L_{1} / L_{0}\right)$ for all solutions for each series of the simulation, we found a best solution in which $\ln \left(L_{1} / L_{0}\right)$ is largest and which satisfied condition $N_{0}=N_{1}$ or $N_{1}>N_{2}$.
Hereafter, we carried out the simulation changing minimum magnitude, $M_{0}$. Three cases, $M_{0}=7.5,7.3$, and 7.0, were considered.

Firstly, we analyze 8 earthquakes of which magnitude are greater than or equal to $7.5\left(M_{0}=7.5\right)$. Since $M \geq 7.5$ earthquakes caused severe damages and were well documented, their magnitudes and focal depths are more reliable than smaller earthquakes. We assumed that all 8 earthquakes occurred in the seismic active periods (that is, $N_{0}=N_{1}$ ). This case corresponds to the example in Section 4 and is shown in Fig. 5. We applied the simulation for the actual observed data. The largest value of $\ln \left(L_{1} / L_{0}\right)$ of the actual data is 7.95. We call this solution Solution 1, which is the same solution shown in Section 4. Note that $T_{P}=100 \mathrm{y}$ is not assumed but is the optimal solution in this case. The optimal velocity is $0.70 \leq V \leq 0.95$. Then, we have simulated 1,000 series of earthquake sequences by using random numbers. For each series, we found a best solution in which the value of $\ln \left(L_{1} / L_{0}\right)$ is largest. Then, we compared the $\ln \left(L_{1} / L_{0}\right)$ values of actual data and those of the simulation. The largest value of $\ln \left(L_{1} / L_{0}\right)$ of the actual data is equal to 7.95 and is the 150th largest $\ln \left(L_{1} / L_{0}\right)$ among the 1,000 series. This simulation is called Simulation 1, in which 372 among the 1,000 series of simulation have no solutions that satisfy all conditions. 
(a)

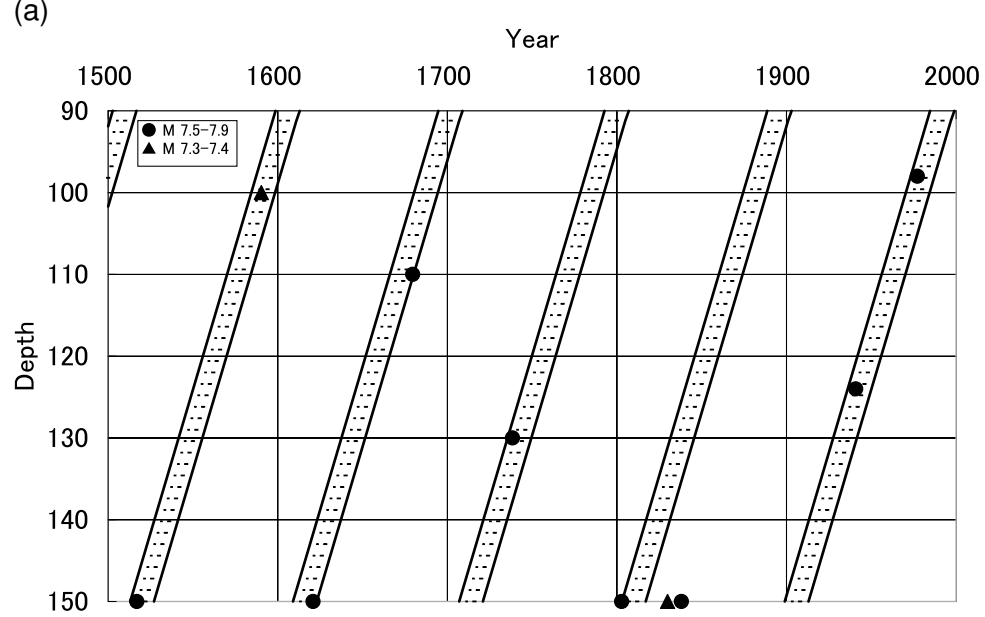

(b)

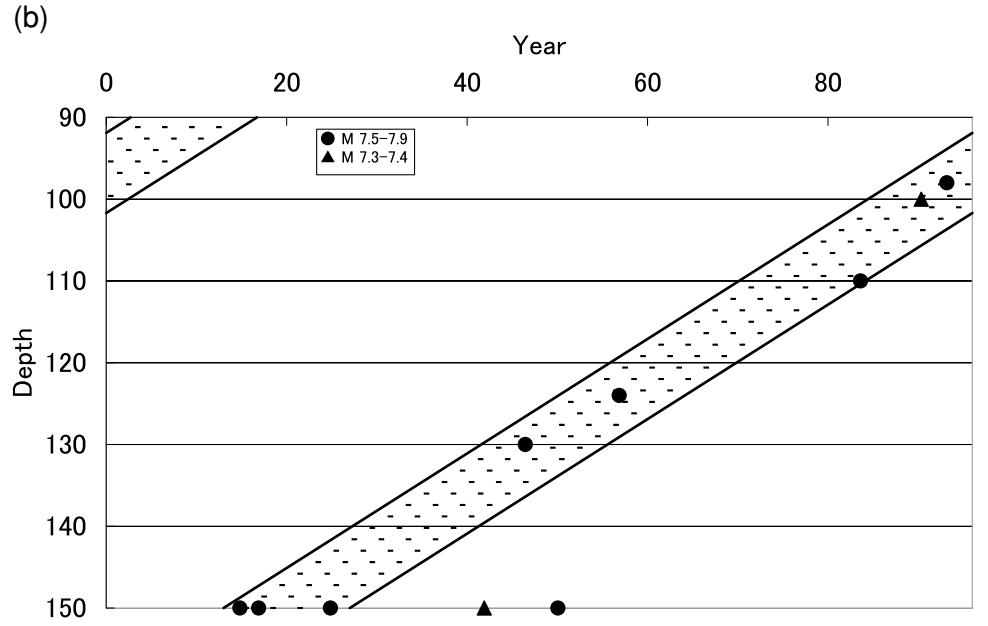

Fig. 6. (a) Solutions 2 and 8 of the statistical PUM model for $M \geq 7.5$ and $M \geq 7.3$ earthquakes excluding a fake $M 7$ earthquake, respectively, in the case of $N_{1}>N_{2}$ (Table 2). (b) Superposed PUM model, in which $T_{P}=96$ years. The symbols are the same as in Fig. 5.

In the same manner, we assume $N_{1}>N_{2}$ instead of $N_{0}=N_{1}$ for $M_{0}=7.5$. The solution is called Solution 2 . The largest value of $\ln \left(L_{1} / L_{0}\right)$ of the actual data is 10.72 and is the 46th largest $\ln \left(L_{1} / L_{0}\right)$ among the 1,000 series. Results are shown in Fig. 6 and Table 2. These results indicate that the PUM model is statistically significant at the $5 \%$ level.

Secondly, we analyze 11 earthquakes of which magnitude are greater than or equal to $7.3\left(M_{0}=7.3\right)$. Results are shown in Table 2. Solutions 3 and 4 indicate solutions when $N_{0}=N_{1}$ and $N_{1}>N_{2}$, respectively. The largest values of $\ln \left(L_{1} / L_{0}\right)$ of the actual data are 10.93 in the both cases and are the 17 th and 74 th largest $\ln \left(L_{1} / L_{0}\right)$ among the 1,000 series, respectively. We call these simulations Simulations 3 and 4, respectively. With regard to Simulation 3, 771 among the 1,000 series of simulation have no solutions that satisfy all conditions. Results are shown in Table 2. These results for Solution 3 also indicate that the PUM model is statistically significant at the $5 \%$ level.

Thirdly, we analyze 24 earthquakes of which magnitude are greater than or equal to $7.0\left(M_{0}=7.0\right)$. Results are shown in Table 2. Solutions 5 and 6 indicate solutions when $N_{0}=N_{1}$ and $N_{1}>N_{2}$, respectively. There is no solution when $N_{0}=N_{1}$. The $\ln \left(L_{1} / L_{0}\right)$ value for Solution 6 is the 941 st largest $\ln \left(L_{1} / L_{0}\right)$ among the 1,000 series. Therefore, the PUM model for $M_{0}=7.0$ is not significant.

\section{Fake $M 7$ Earthquakes and New Dataset}

The PUM model could explain the behavior of the $M \geq 7.3$ earthquakes that have occurred during the past 500 years, as shown in Figs. 5 and 6, and Table 2. However, some earthquakes including many of the $7.0 \leq M<7.3$ earthquakes do not conform to the model. Let us investigate the cause of nonconformity. There are 5 pairs of earthquakes in the case of which the time interval between the 2 earthquakes in a given pair is less than 5 years, as shown in Table 1: these pair are $(1543,1545),(1590,1595),(1679$, $1681),(1738,1740)$, and $(1893,1894)$. The interval between the earthquakes in 4 pairs is 1 or 2 years and that of 1 pair is 5 years and is nearly integer. The time intervals $(d T)$ between the earthquakes in all the 5 pairs are also listed in Table 1.

In order to verify the reliability of these paired earthquakes, we compared the data given in the ROMPLUS catalog with those in the other available catalogs (Table 1). We used the following data: (1) data from Utsu's Cata$\log$ of Damaging Earthquakes in the World (Utsu, 1990, 1992; International Institute of Seismology and Earthquake Engineering, 2006); (2) data from the catalog of strong intermediate earthquakes (1100-1973) occurred in 


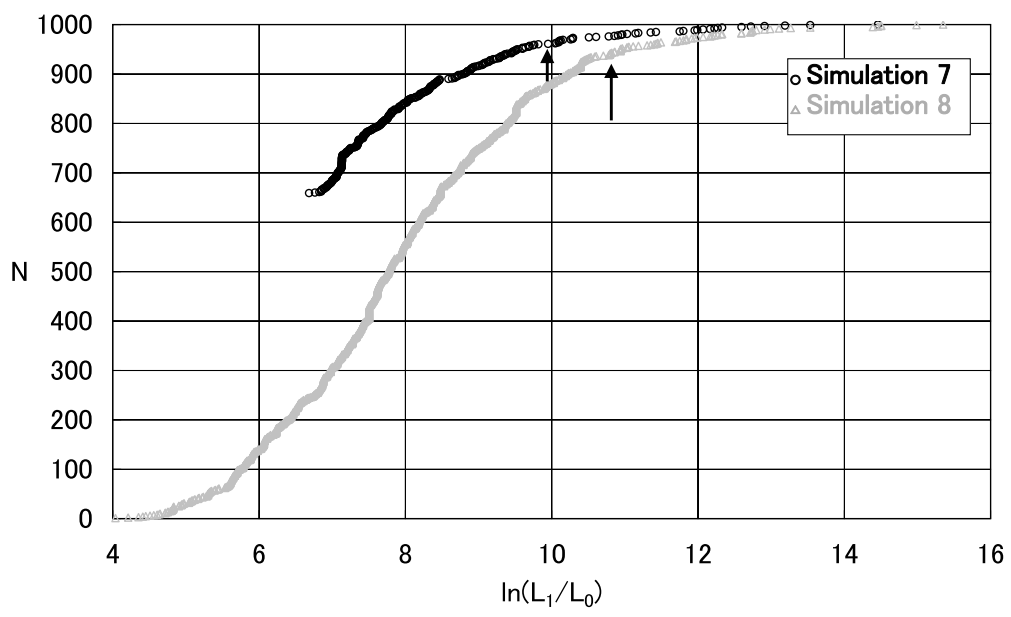

Fig. 7. Cumulative distribution of $\ln \left(L_{1} / L_{0}\right)$ obtained by 1,000 series of simulations 7 and 8 for the $M \geq 7.3$ earthquakes. The arrows indicate $\ln \left(L_{1} / L_{0}\right)$ of solutions 7 and 8 of actual data shown in Table 2 .

the Vrancea-Carpathian region (Purcaru, 1979); (3) the data on earthquakes with MSK intensity $I_{0} \geq 7$ occurred during 1471-1970 (Enescu et al., 1974); and (4) data from Radu's catalog of major historical Vrancea earthquakes (the maximum MSK intensity $I_{0} \geq 8$ ) (Lungu and Aldea, 2001). Although these catalogs depend on mutually, each catalog was made based on authors' own ideas. Regarding to these 5 pairs of earthquakes, one of the paired earthquakes is not listed in 2 or more different catalogs except the (1679, 1681) pair. Among the 24 earthquakes listed in Table 1, the following 4 earthquakes are not listed in Utsu's cata$\log$ : the 1543, 1595, 1740, and 1893 earthquakes. Each of these earthquakes is a part of above mentioned paired earthquakes. These earthquakes are not listed in Purcaru's catalog, too. Although Radu's catalog does not list the first 2 earthquakes, it lists the last 2 earthquakes. However, the Gutenberg-Richter magnitudes of the last 2 earthquakes estimated by Radu are 5.8 and 5.7. Therefore, we conclude that the $1543,1595,1740$, and 1893 earthquakes with $M \geq 7.0$ are fake $M 7$ earthquakes. The main reason why they are fake $M 7$ earthquakes will be overestimation of magnitudes.

We suppose that in the ROMPLUS catalog, the same earthquake has been listed twice with different origin times and hypocentral parameters or the $M$ value of small earthquakes have been overestimated. Therefore, we conclude that earthquake no. 2, 6, 14, and 19 listed in Table 1 are fake $M 7$ earthquakes; therefore, we exclude them from our analyses. These 4 fake $M 7$ earthquakes are indicated with mark "F" in Figs. 4 and 5.

The new dataset that excludes 4 fake $M 7$ earthquakes is called Dataset 2, while the original dataset that includes all $24 M \geq 7.0$ earthquakes is called Dataset 1 .

We carried out same simulation for Dataset 2 as for Dataset 1. Results are summarized in Table 2. Since there is no $M \geq 7.5$ fake earthquake, cases in $M_{\mathrm{o}}=7.3$ and $M_{\mathrm{o}}=7.0$ are shown. Solutions 7 and 8 indicate solutions when $N_{0}=N_{1}$ and $N_{1}>N_{2}$ for $10 M \geq 7.3$ earthquakes. Since optimal parameters in Simulations 7 and 8 are same as those in Simulations 1 and 2, solutions and data are shown in Figs. 5 and 6, respectively, too. Then, we have simulated 1,000 series of earthquake sequences by using random numbers. For each series, we found a best solution in which the value of $\ln \left(L_{1} / L_{0}\right)$ is largest. Then, we compared the $\ln \left(L_{1} / L_{0}\right)$ values of actual data and those of the simulation. We call these simulations Simulations 7 and 8 , respectively. The largest values of $\ln \left(L_{1} / L_{0}\right)$ of the actual data in both solutions are 9.94 and 10.82 and are the 41 st and 58th largest $\ln \left(L_{1} / L_{0}\right)$ among the 1,000 series, respectively. These results for Solution 7 also indicate that the PUM model is statistically significant at the 5\% level. The cumulative distribution of largest $\ln \left(L_{1} / L_{0}\right)$ for 1,000 series for Simulations 7 and 8 are shown in Fig. 7. With regard to Simulation 7, 657 among the 1,000 series of simulation have no solutions that satisfy all conditions.

Solutions 9 and 10 indicate solutions when $N_{0}=N_{1}$ and $N_{1}>N_{2}$ for $20 M \geq 7.0$ earthquakes, respectively. In the case of $N_{0}=N_{1}$, there is no solution, while in the case of $N_{0}>N_{1}$, the largest value of $\ln \left(L_{1} / L_{0}\right)$ of the actual data in this solution is 7.98 and is the 288th largest $\ln \left(L_{1} / L_{0}\right)$ among the 1,000 series. Although these results indicate that the PUM model is not statistically significant, data fitting to the PUM model was improved largely comparing results using Dataset 1 that include 4 fake $M 7$ earthquakes.

The repetition period, $T_{P}$, of all optimal solutions shown in Table 2 except Solution 6 ranges from 93 and 100 years, which is nearly one century.

\section{Discussions}

\subsection{Geometry of earthquake sequences in Vrancea}

We have demonstrated that the PUM model is better than the Poisson model for $M \geq 7.5$ and $M \geq 7.3$ earthquakes. However, this is not the case for $M \geq 7.0$ earthquakes. We suppose that this difference between $M \geq 7.3$ and $M \geq 7.0$ earthquakes can be attributed to the errors in their focal depths. The focal depths of small earthquakes determined from intensity distributions contain larger errors. On the basis of these observations and results of simulation, we propose the following regularity for Vrancea intermediatedepth earthquakes. (1) The first $M 7$ earthquake ( $M=$ 7.1-7.9) occurs in a deep segment (depth: 140-160 km) of seismic regions at the beginning of each century (1-16 


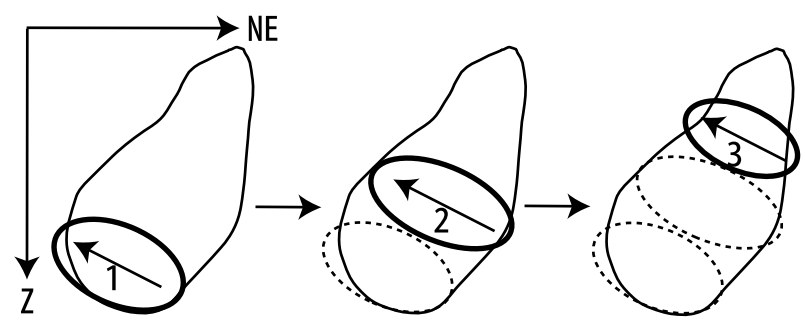

Fig. 8. Schematic diagram of model for the $M 7$ earthquakes occurring during a century.

years). (2) The second $M 7$ earthquake ( $M=7.1-7.7)$ occurs in a midst segment (depth: $110-140 \mathrm{~km})$ of seismic regions in the middle of each century (37-45 years). (3) The third $M 7$ earthquake $(M=7.1-7.5)$ occurs in a shallow segment (depth: $80-110 \mathrm{~km}$ ) of seismic regions at the end of each century (77-94 years). This migration phenomenon repeats every century. The data given in parentheses are actual data on earthquakes that have occurred during the last 500 years which is shown in Table 1 .

Figure 8 shows the schematic of the proposed model. Three $M 7$ earthquakes occur every 100 years and release stress inside seismic regions. Since the uppermost region is too narrow to generate an $M 7$ earthquake, an $M 6.9$ earthquake that occurred in 1990 is the largest earthquake. Note that $M 7$ earthquakes can sometimes occur irregularly, such as the $M 7.2$ earthquake that occurred in 1986 .

The rupture of each earthquake is unilateral; it starts at the NE edge and propagates toward the SW (Muller et al., 1978; Fuchs et al., 1979; Hartzell, 1979; Rakers and Muller, 1982; Iosif et al., 1983; Hurukawa et al., 2008). The focal mechanism of the Vrancea intermediate-depth earthquakes indicates down-dip extension and thrust with NW-SE $P$ axes. Since no plate boundary is present, paleo-subduction zones are assumed to be present (e.g., Fuchs et al., 1979; Sperner et al., 2004). Therefore, the negative buoyancy of the remnant of an old plate is considered to be the cause of these earthquakes. The PUM model proposes that the accumulated stress inside the old plate is released from the bottom to the top in turn, and this process is repeated periodically without a long period of quiescence. This fact may indicate that the origin of the stress source is situated at the bottom of the seismic regions and the negative buoyancy of the subducting slab is the main source of the stress. Since the proposed model shows how intermediate-depth earthquakes occur in the Vrancea region, this model can be used for understanding why these earthquakes occur.

\subsection{Next $M 7$ earthquakes}

We have confirmed that the repetition period of earthquakes is approximately 100 years. This implies that seismic activities may take place in this century, too. In order to predict future earthquakes, we analyzed earthquakes that occurred in each seismic active period (indicated by the hatched regions in Fig. 9), because the errors in the focal depths of old $7.0 \leq M<7.3$ earthquakes lead to the degradation of data fitting. We assumed the repetition period of earthquakes is 100 years for simplicity.

Figure 9 shows the relationship between the actual year and the year of each century in which $M \geq 7.0$ earthquakes

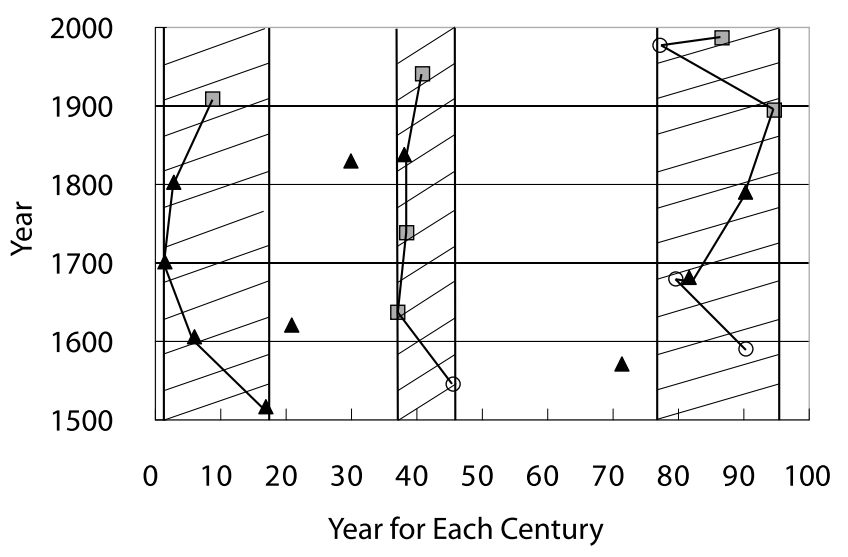

Fig. 9. Relationship between the actual year and year for each century in which $M \geq 7.0$ earthquakes occurred during 1500-2000. Data is obtained from ROMPLUS, except those for the 4 fake $M 7$ earthquakes. The hatched regions indicate high-seismicity periods. Earthquakes repeated in the same segment have been connected by lines. Open circle, solid square and solid triangle indicate earthquakes with depths of 100-120, 120-140, and 140-150 km, respectively.

Table 3. Hypocenter parameters of the $3 M 7$ earthquakes expected to occur in the 21 st century.

\begin{tabular}{cccrc}
\hline Year & Lat. & Lon. & $Z, \mathrm{~km}$ & $M$ \\
\hline $2007.2 \pm 5.5$ & $45.6 \pm 0.2$ & $26.6 \pm 0.2$ & $150 \pm 10$ & $7.3 \pm 0.3$ \\
$2040.0 \pm 3.0$ & $45.8 \pm 0.1$ & $26.9 \pm 0.1$ & $124 \pm 10$ & $7.4 \pm 0.3$ \\
$2086.8 \pm 6.4$ & $45.9 \pm 0.1$ & $27.0 \pm 0.1$ & $98 \pm 10$ & $7.2 \pm 0.2$ \\
\hline
\end{tabular}

occurred during 1500-2000. Using 5 or 7 earthquakes, connected by solid lines in this figure, occurring in each of the 3 seismic active time periods (indicated by the hatched areas in Fig. 9), we obtain the average year and the standard deviation of year of occurrence of earthquakes. They are $7.2 \pm 5.5,40.0 \pm 3.0$, and $86.8 \pm 6.4$. Thus, 3 earthquakes can be expected to occur in the 21 st century. The magnitude of these earthquakes can be calculated from the averages values of $M$ of these earthquakes in the same manner. On the other hand, the hypocenters of these earthquakes can be estimated on the basis of those of the 1940 and 1977 earthquakes and those of the earthquakes listed in Table 1. On the basis of the pattern of occurrence of historical earthquakes, we can assume that $3 M 7$ earthquakes may occur in the 21 st century in the years $2007.2 \pm 5.5,2040.0 \pm 3.0$, and $2086.8 \pm 6.4$. Hypocenter parameters of the $3 M 7$ earthquakes expected to occur in the 21 st century are listed in Table 3.

Furthermore, by referring to the source processes of the recent large earthquakes, the source processes of future earthquakes can be predicted as follows. (1) A pure thrust exists with a strike of NE-SW, and the steep-dip nodal plane dips toward the NW with an angle of $60^{\circ}-80^{\circ}$. (2) The rupture is unilateral, and it propagates from the NE toward the SW. (3) The length of the fault is $30-50 \mathrm{~km}$.

In this study, we could easily identify 3 seismic activities at different focal depths according to the PUM model. Although by using Solutions 2, 4, 8, and 10, some earthquakes have been found to occur during the low-seismicity periods, 
we suppose that this inconsistency was caused by errors in their focal depths. In the PUM model, the number of inconsistent events is greater for $7.0 \leq M<7.3$ earthquakes than for $M \geq 7.3$ earthquakes. This inconsistency can be attributed to lack of availability of suf cient intensity data on small earthquakes resulting in the introduction of errors in the estimated focal depths; further, the estimated errors in focal depths are large in the case of small earthquakes than those in the case of large earthquakes. However, since stress is accumulated and released in a constant manner in seismic regions, it can be considered that almost all $M 7$ earthquakes occur as predicted by the PUM model. This is consistent with the fact that all $M 7$ earthquakes predicted by the model have occurred as shown in Fig. 9, with in a maximum depth error of $50 \mathrm{~km}$.

\section{Conclusion}

We analyzed the data on $M \geq 7.0$ intermediate-depth earthquakes that occurred in the Vrancea region of Romania during the period 1500-2000 from ROMPLUS, a Romanian earthquake catalog. On the basis of this analysis, we proposed the periodic upward migration (PUM) model. According to this model (1) the rst $M 7$ earthquake occurs in a deep segment (depth: 140-160 km) of seismic regions at the beginning of each century, (2) the second $M 7$ earthquake occurs in a midst segment (depth: 110-140 km) of seismic regions in the middle of each century, and (3) the third $M 7$ earthquake occurs in a shallow segment (depth: $80-110 \mathrm{~km}$ ) of seismic regions at the end of each century. This migration phenomenon repeats every century. By using the difference of $\log$-likelihood, $\ln \left(L_{1} / L_{0}\right)$, between the PUM and uniform Poisson models, we could demonstrate that this model is better than a uniform Poisson model with regard to time and space (depth) for $M \geq 7.5$ and $M \geq 7.3$ earthquakes. In order to con $\mathrm{rm}$ the validity of the PUM model, we also carried out the simulation by producing random numbers using the bootstrap method, in which origin times and depth distribution for observed earthquakes were preserved. We carried out the simulation changing minimum magnitude, $M_{0}$. Three cases, $M_{0}=7.5,7.3$, and 7.0, were considered. The results of the simulation for $M \geq 7.5$ and $M \geq 7.3$ indicated that the PUM model is signi cant at the $5 \%$ level. The next $M 7$ earthquake is expected to occur at a depth of 140-160 km at the beginning of this century. The source processes of future earthquakes can be predicted as follows. (1) A pure thrust exists with a strike of NE-SW, and a steep-dip nodal plane dips toward the NW with an angle of $60^{\circ}-80^{\circ}$. (2) The rupture is unilateral and it propagates from the NE to the SW. (3) The length of the fault is $30-50 \mathrm{~km}$.

Acknowledgments. We thank Dr. Mihaela Popa for providing us with the ROMPLUS catalog and Dr. Emil-Sever Georgescu for his valuable discussions on data on historical earthquakes. We also thank anonymous reviewers for their constructive comments.

\section{References}

Constantinescu, L. and V. I. Marza, A computer-compiled and computer- oriented catalog of Romania's earthquakes during a millennium (AD984-1979), Rev. Roum. Geophys., 24, 193-234, 1980.

Dziwonski, A. M., T.-A. Chou, and J. Woodhouse, Determination of earthquake source parameters from waveform data for studies of global and regional seismicity, J. Geophys. Res., 86, 2825-2852, 1981.

Enescu, D., V. Marza, and I. Zamarca, Contributions to the statistical prediction of Vrancea earthquakes, Rev. Roum. Geophys., 18, 67, 1974. Frohlich, C., Deep Earthquakes, Cambridge Univ. Press, 2006.

Fuchs, K., K.-P. Bonjer, G. Bock, I. Cornea, C. Radu, D. Enescu, D. Jianu, A. Nourescu, G. Merkler, T. Moldoveanu, and G. Tudorache, The Romanian earthquake of March 4, 1977, II. Aftershocks and migration of seismic activity, Tectonophysics, 53, 225-247, 1979.

Hartzell, S., Analysis of the Bucharest strong ground motion record for the March 4, 1977 Romanian earthquake, Bull. Seismol. Soc. Am., 69, 513-530, 1979.

Hurukawa, N., M. Popa, and M. Radulian, Relocation of large intermediate-depth earthquakes in the Vrancea region, Romania, since 1934 and a seismic gap, Earth Planets Space, 60, 565-572, 2008.

Imoto, M., Changes in seismicity of microearthquakes before major earthquakes in the Kanto area, Zisin, 45, 177-185, 1992 (in Japanese with English abstract).

Imoto, M. and N. Hurukawa, Assessing potential seismic activity in Vrancea, Romania, using a stress-release model, Earth Planets Space, 58, 1511-1514, 2006.

International Institute of Seismology and Earthquake Engineering, Catalog of damaging earthquakes in the world, http://iisee.kenken.go.jp/utsu/index_eng, Cited 16 Feb 2006, 2006.

Iosif, T., M. C. Oncescu, and S. Iosif, March 4, 1977 Vrancea earthquake, spatial distribution of events and temporal evolution of focal mechanism, Bull. Int. Inst. Seismol. Earthq. Eng., 20, 1-18, 1983.

Lungu, D. and A. Aldea, Radu catalogue of major historical Vrancea earthquakes (maximum MSK intensity, $I_{0} \geq 8$ ), in Earthquake Hazard and Countermeasures for Existing Fragile Buildings, edited by Lungu, D. and Saito, T., 293-294, 2001.

Mogi, K., Relationship between shallow and deep seismicity in the western Paci c region, Tectonophysics, 17, 1-22, 1973.

Mogi, K., Recent seismic activity in the Tokai (Japan) region where a large earthquake is expected in the near future, Tectonophysics, 138, 255-268, 1987.

Muller, G., K.-P. Bonjer, H. Stockl, and D. Enescu, The Romanian earthquake of March 4, 1982, I. Rupture process inferred from fault-plane solution and multiple-event analysis, J. Geophys., 44, 203-218, 1978.

Oncescu, M., V. I. Marza, M. Rizescu, and M. Popa, The Romanian earthquake catalogue between 984-1997, in Vrancea Earthquakes: Tectonics, Hazard and Risk Mitigation, edited by Wenzel, F. et. al., 43-47, 1999.

Purcaru, G., Quasi- and supercyclicity of earthquakes and time-magnitude gaps in earthquake prediction, Tech. Rep. NORSAR Sci. Rep., No. 673/74, 53-55, 1974.

Purcaru, G., The Vrancea, Romania, earthquake of March 4, 1977-A quite successful prediction, Phys. Earth Planet. Inter., 18, 274-287, 1979.

Rakers, E. and G. Muller, The Romanian earthquake of March 4, 1977, III. Improved focal model and moment determination, J. Geophys., 50, 143-150, 1982.

Sperner, B., D. Ioane, and R. J. Lillie, Slab behaviour and is surface expression: new insights from gravity modeling in the SE-Carpathians, Tectonophysics, 382, 51-84, 2004.

Utsu, T., Correlation between shallow earthquakes in Kwanto region and intermediate earthquakes in Hida region, central Japan, Zisin, 1975 (in Japanese with English abstract).

Utsu, T., Catalog of Damaging Earthquakes in the World (from Ancient to 1989), 1990 (in Japanese).

Utsu, T., Destructive earthquakes in the world, 1500-1992, Earthquake Disaster Reduction Handbook, Building Research Institute, 1-24, 1992.

Utsu, T., Seismicity Studies: A Comprehensive Review, Uni. Tokyo Press, 1999 (in Japanese).

Wenzel, F., D. Lungu, and O. Novak, (eds), Vrancea Earthquakes: Tectonics, Hazard and Risk Mitigation, Kluwer Academic Publishers, 1999.

N. Hurukawa (e-mail: hurukawa@kenken.go.jp) and M. Imoto 